\title{
Thread-based bioluminescent sensor for detecting multiple antibodies in a single drop of whole blood
}

Kosuke Tomimuro ${ }^{l}$, Keisuke Tenda ${ }^{l}$, Yan $\mathrm{Ni}^{2}$, Yuki Hiruta ${ }^{1}$, Maarten Merkx ${ }^{1,2 *}$, Daniel Citterio ${ }^{1 *}$

${ }^{1}$ Department of Applied Chemistry, Keio University, 3-14-1 Hiyoshi, Kohoku-ku, Yokohama 223-

8522, Japan.

${ }^{2}$ Laboratory of Chemical Biology and Institute for Complex Molecular Systems, Department of Biomedical Engineering, Eindhoven University of Technology, P.O. Box 513, 5600 MB

Eindhoven, The Netherlands

*To whom correspondence should be addressed.Email: citterio@applc.keio.ac.jp; m.merkx@tue.nl 


\section{Table of Contents}

Figure S1 Illustration of sensor protein structure and working mechanism

Figure S2 Fabrication steps of $\mu$ TADs (final design)

Figure S3 Photographs of setups used for capturing bioluminescence

Figure S4 Photograph and design of 3D-printed case

Figure S5 Procedures of data processing for obtaining hue $(\mathrm{H})$ and value $(\mathrm{V}) \quad$ S7

Figure S6 Fabrication steps of $\mu$ TADs, signaling single emission S8

Figure S7 Influence of thread twisting turns on hue signal S9

Figure S8 Illustration of detailed sewing patterns of alternative $\mu$ TADs

S10

Figure S9 Illustration of the detailed vertical structure of $\mu$ TADs

S11

Figure S10 Results of reproducibility evaluation

Figure S11 Results of storage stability evaluation

Equation S1 Equation used for fitting the calibration curves

Table S1 Analytical parameters obtained from Figures 3A and S8

Table S2 Analytical parameters obtained from Figures 6A and 7A

References 
a)

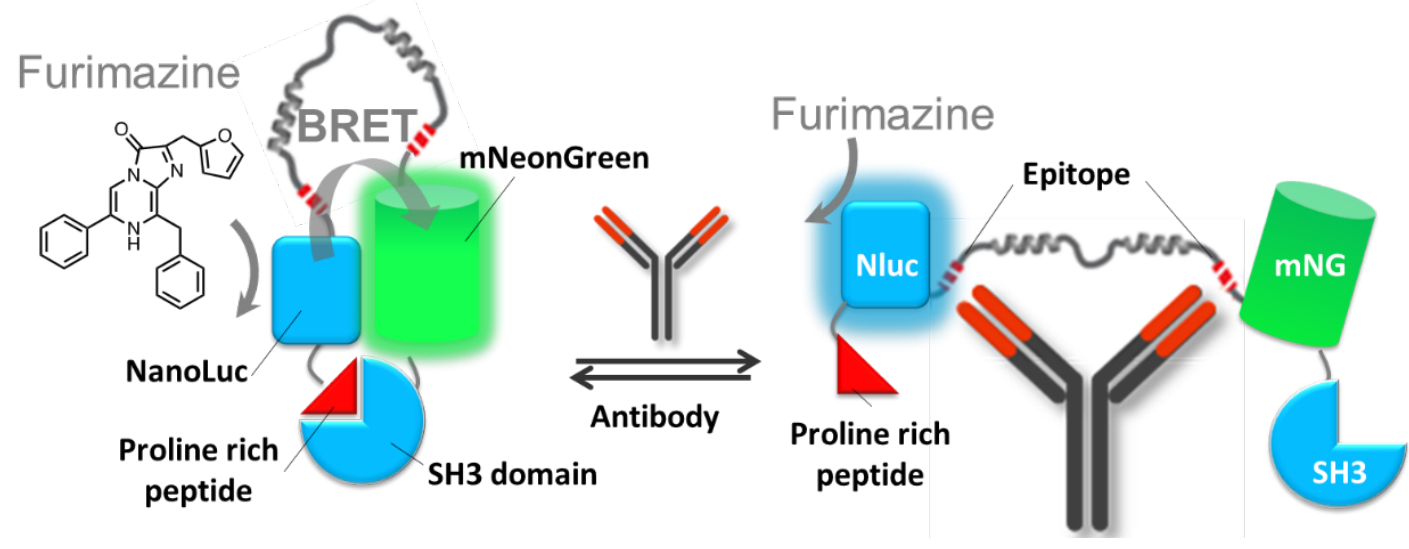

b)
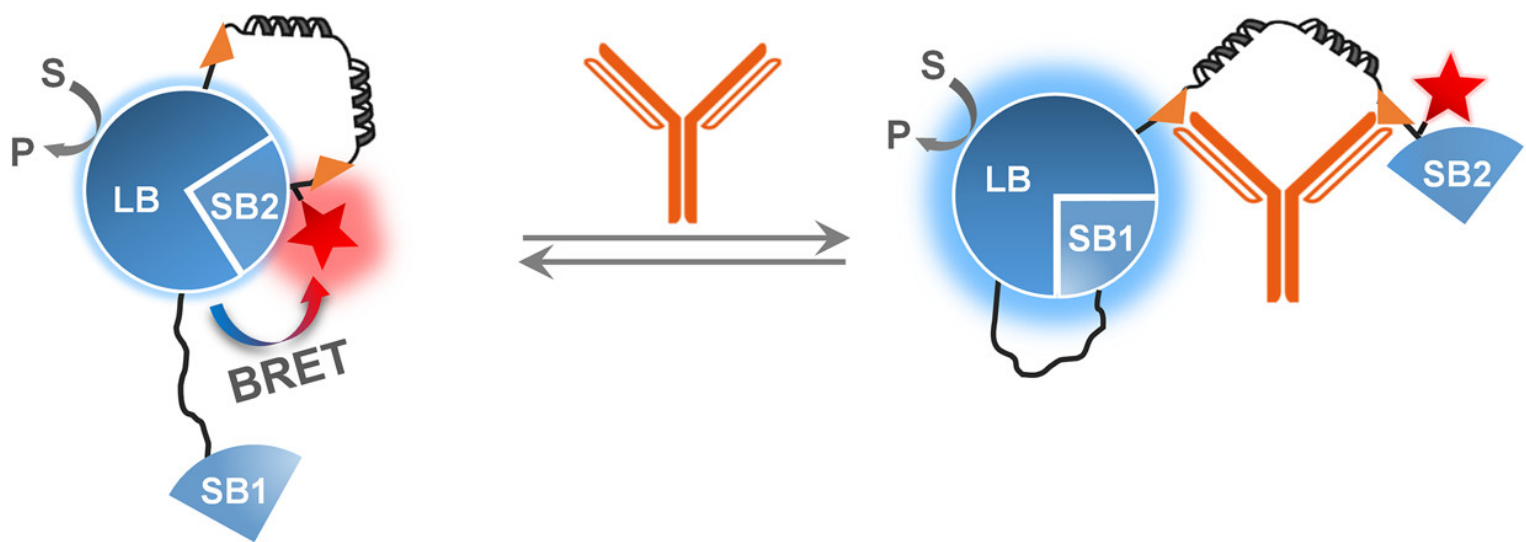

Figure S1. Schematic illustration of the sensor protein structure and its working principle ${ }^{1,2}$

a) Conventional LUMABS: In the absence of the targeted antibody, the blue light emitting luciferase (NanoLuc; Nluc) and the green fluorescent protein (mNeonGreen; $\mathrm{mNG}$ ) are located in close proximity, resulting in efficient bioluminescence energy transfer (BRET) and hence, green light emission upon luciferase catalyzed oxidation of the luciferin (furimazine). On the other hand, the bivalent binding of the targeted antibody to the epitope sequences breaks the link between the proline rich peptide and SH3 helper domains. This results in spatial separation of the luciferase and the green fluorescent protein, preventing the BRET and hence, shifting the emitted light color from green to blue. Adapted with permission from Arts, R.; den Hartog, I.; Zijlema, S. E.; Thijssen, V.; van der Beelen, S. H. E.; Merkx, M. Anal. Chem. 2016, 88, 4525-4532. Copyright 2016 American Chemical Society.

b) NB-LUMABS: In the absence of target antibody, the large bit (LB) and the Cy3-conjugated small bit 2 (SB2) fragments are combined into the complemented Nanoluc luciferase, resulting in efficient BRET and hence, red light emission upon oxidation of the furimazine substrate (S). In the presence of the target antibody, the fluorescent dye-conjugated SB2 unit is replaced by the non dye-conjugated small bit 1 (SB1) in a competitive intramolecular rearrangement, preventing the BRET and hence, switching the bioluminescence emission to blue. Reprinted with permission from Ni, Y.; Arts, R.; Merkx, M. ACS Sens. 2019, 4, 20-25. Copyright 2019 American Chemical Society. 
a)

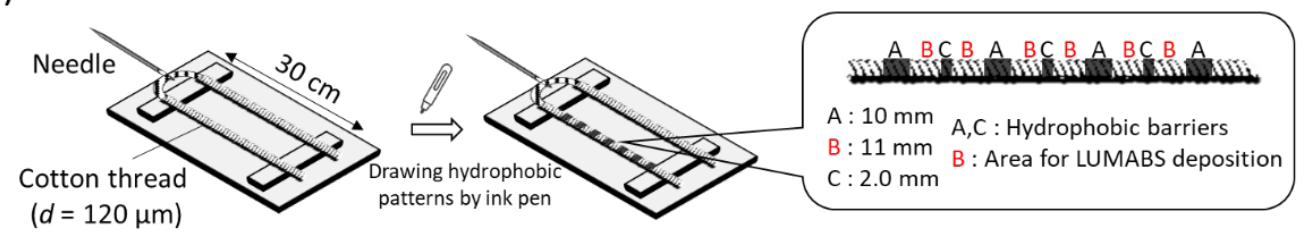

b)

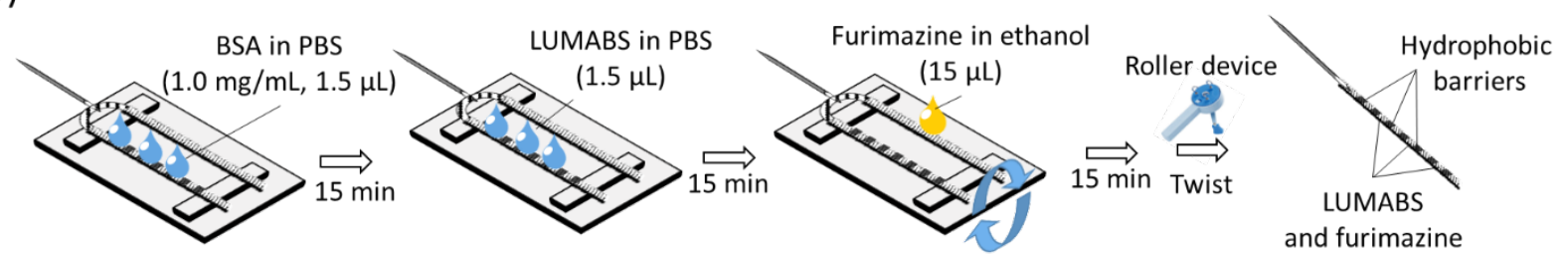

c)

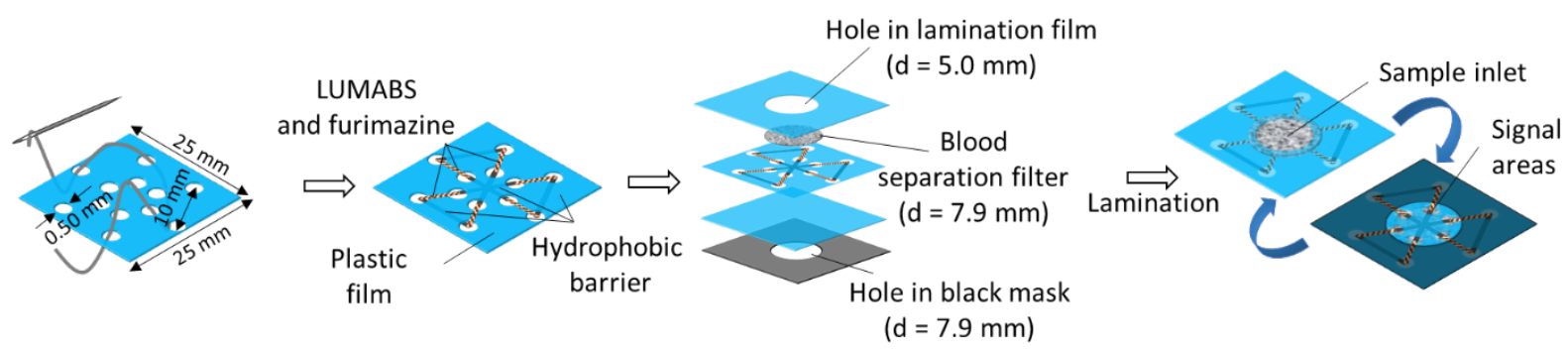

Figure S2. Schematic illustration of fabrication steps for the final construction of $\mu$ TADs: a) Drawing hydrophobic patterns on cotton thread by a permanent ink pen; b) applying solutions containing blocking reagent and stabilizer (BSA), LUMABS (luciferase) and furimazine (luciferin) on different parts of the thread, followed by twisting the two thread arms by the roller device; c) sewing the twisted threads on a plastic film with precut holes, and vertically assembling all layers including lamination films, blood separation filter and a black mask. 
a)

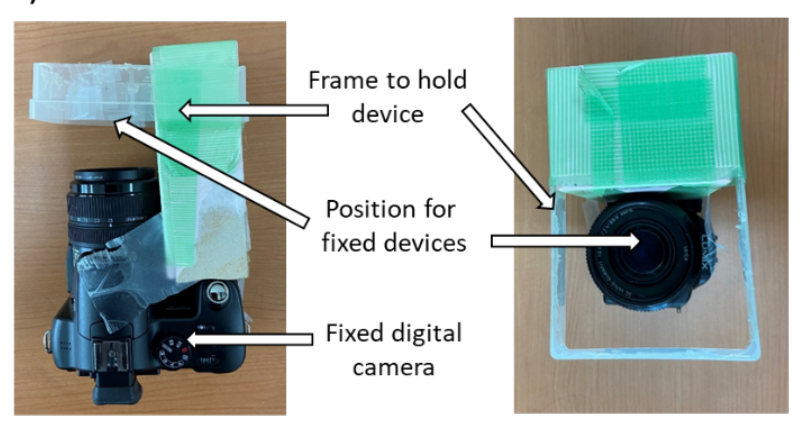

b)

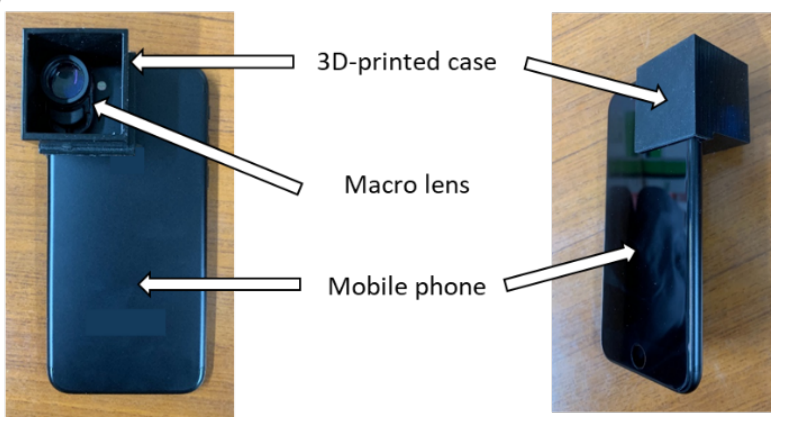

Figure S3. Setups for recording photographs to capture bioluminescence signals: a) by the digital camera; or b) by the camera of a mobile phone. The distances between the camera lens and the device are a) $2.5 \mathrm{~cm}$ and b) $1.5 \mathrm{~cm}$, respectively. 
a)

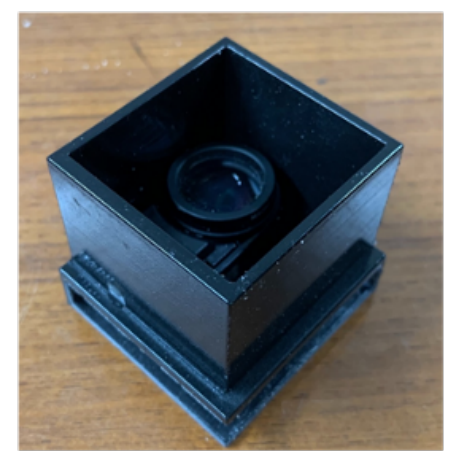

b)

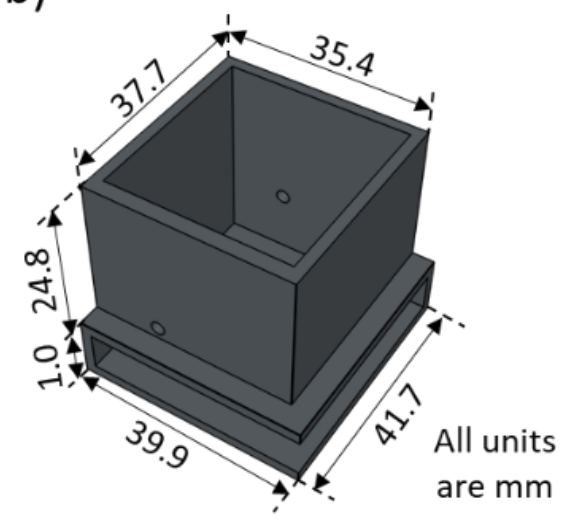

Figure S4. a) Photograph of the 3D-printed case combined with the macro lens; b) design of the 3D-printed case in the Shape3D software. 
(a)

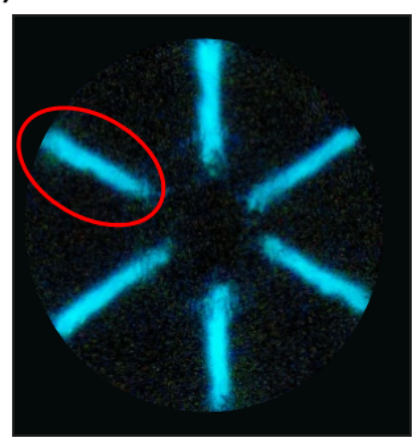

(b)

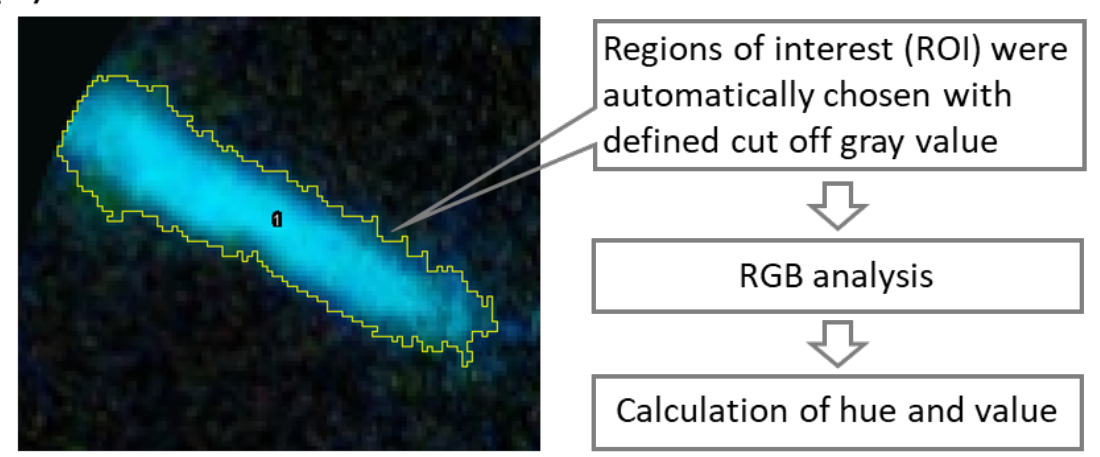

Figure S5. Procedure for data processing to obtain hue and value (HSV color coordinates): a) example image showing light emission from 6 detection areas on a single device; b) magnification of a single detection area with the region of interest automatically selected based on a defined cut off gray value; the hue and the value calculated from RGB values in each detection area were used for data processing. ${ }^{3}$ 

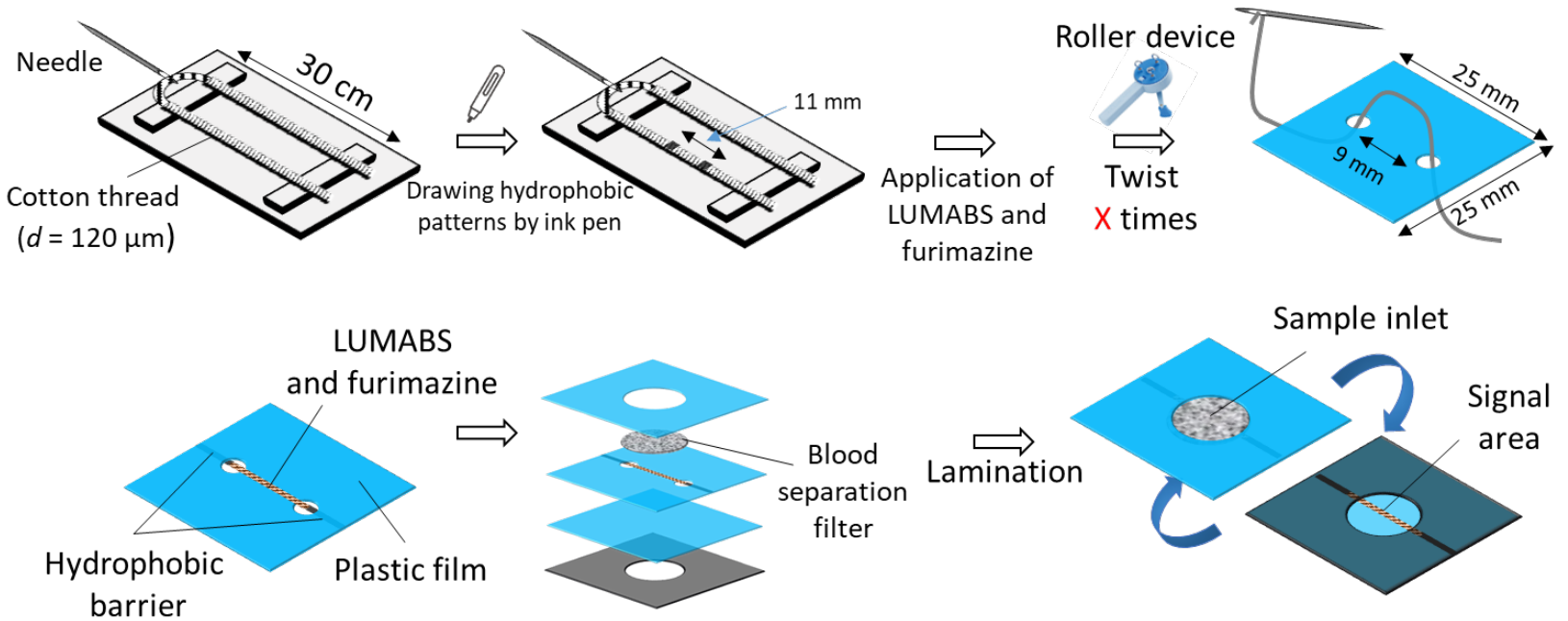

Figure S6. Schematic illustration of fabrication steps for a single signal area $\mu$ TAD used for evaluating the influence of the number of twisting turns on the bioluminescence reaction performed on intertwisted threads. 
a)

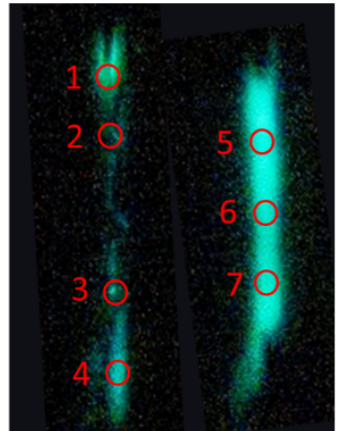

10 turns 60 turns

b)

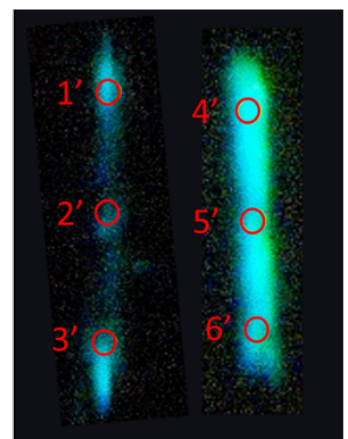

10 turns 60 turns
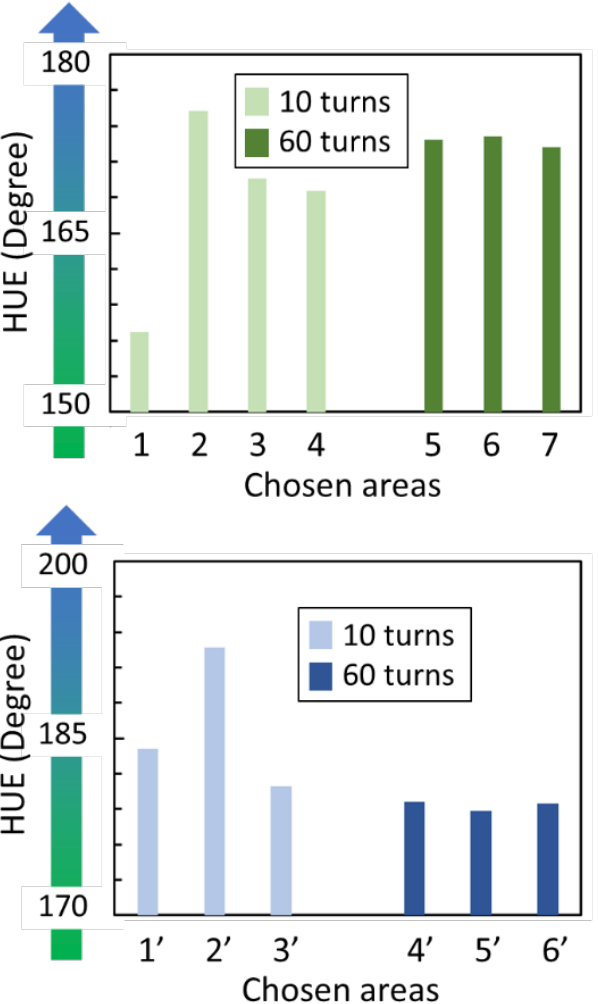

Figure S7. Photographs showing examples of the local bioluminescence emission from intertwisted threads with different twisting turns (10 and 60 turns, respectively) and the corresponding local hue signals for each area of the threads after application of $5 \mu \mathrm{L}$ blood samples spiked with a) $0 \mathrm{nM}$ and b) $100 \mathrm{nM}$ of anti-HIV. 


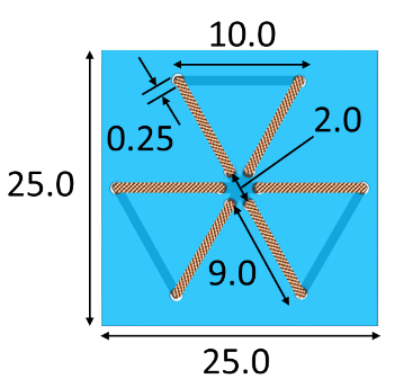

(a)

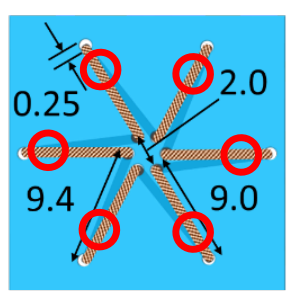

(b)

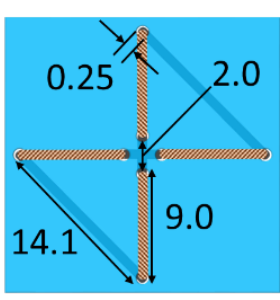

(c)

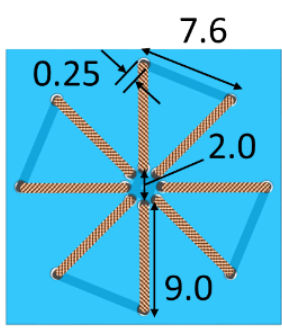

(d)

\section{All units are $\mathrm{mm}$}

Figure S8. Schematic illustration of the detailed sewing patterns (top view) of $\mu$ TAD variations; (a) - (d) refer to the corresponding patterns shown in Fig. $2 \mathrm{~B}$ of the main text; the red circles in pattern (b) indicate the regions where parts of the bioluminescence emitting thread sections are being crossed by non-light emitting sections. 
a)
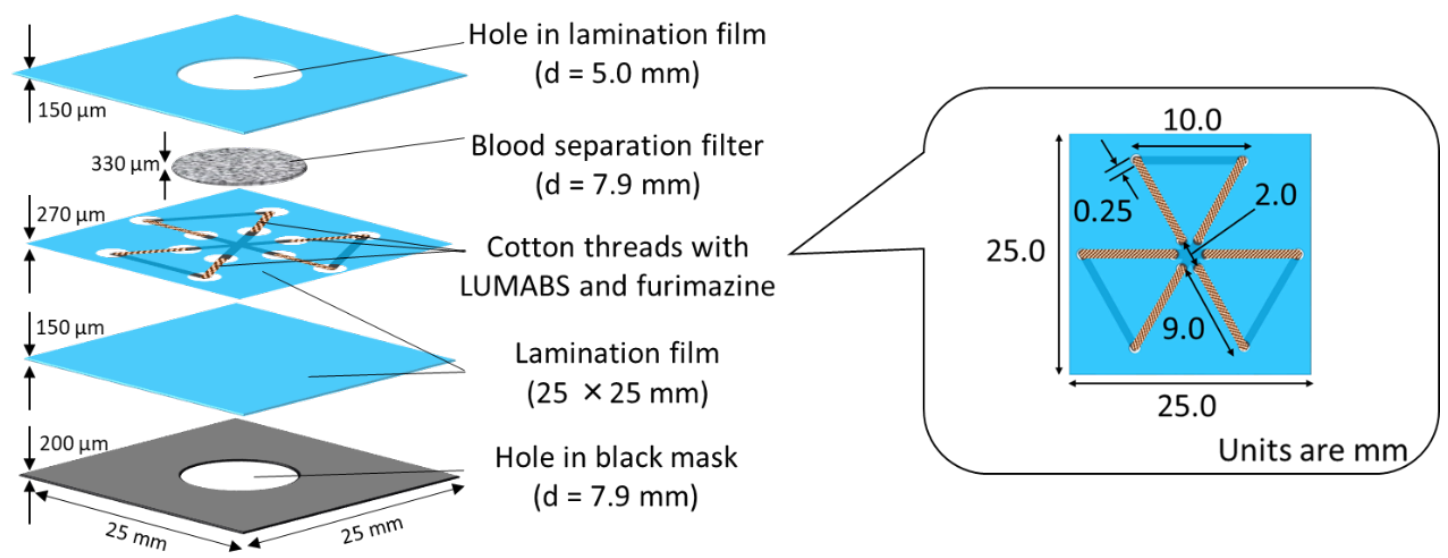

b)
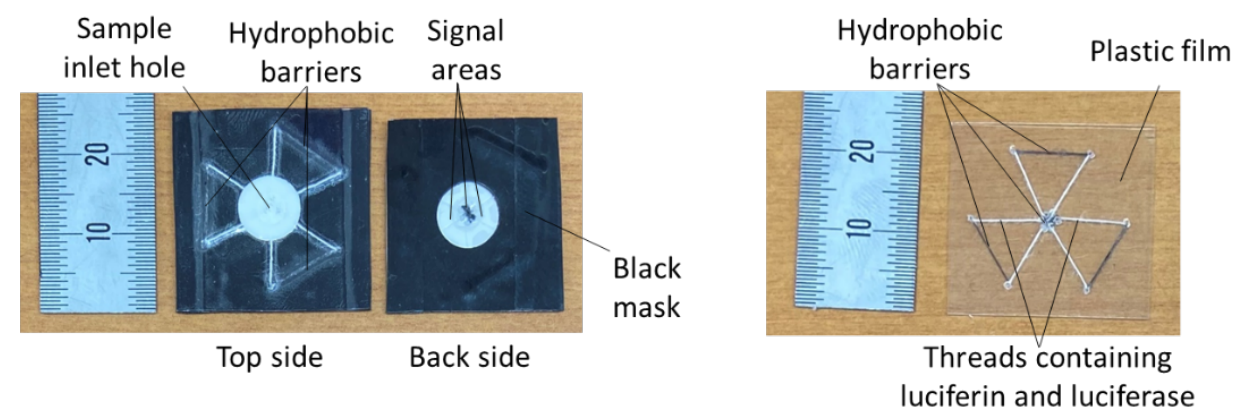

Figure S9. a) Detailed schematic illustration of the optimized vertically assembled $\mu$ TAD together with the sewing pattern on the plastic film (please note that the schematic representation of the sewing pattern is not to scale); b) photographs of the $\mu$ TADs and the sewing pattern on a plastic film. 


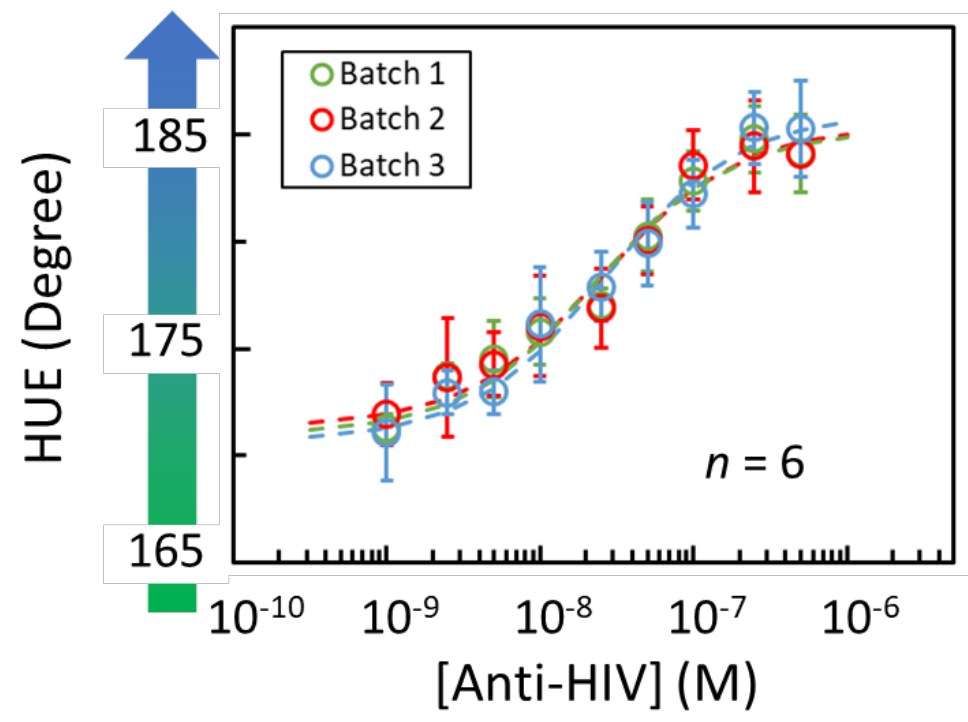

Figure S10. Fabrication reproducibility of three batches of individually fabricated $\mu$ TADs based on HIVLUMABS; plots and error bars represent mean values $\pm 1 \sigma$ obtained from six signal detection areas of a single device. 

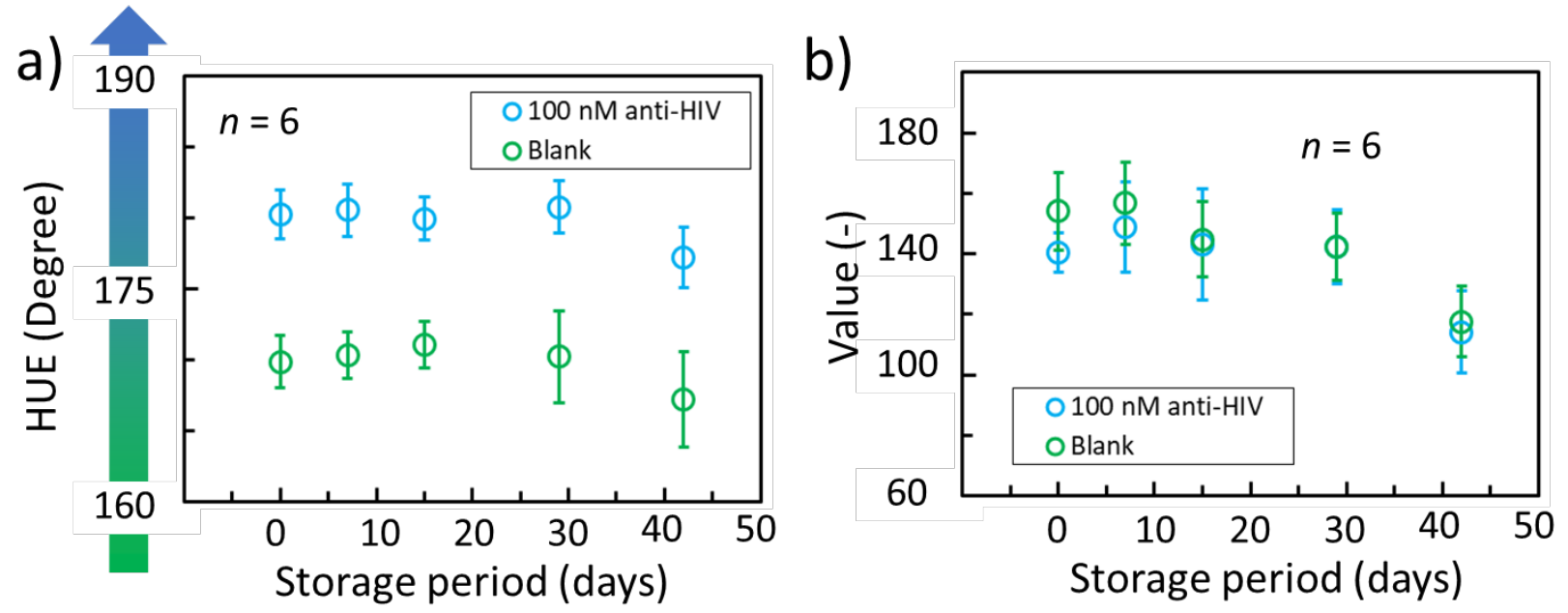

Figure S11. Results of storage stability evaluation of anti-HIV targeting $\mu$ TADs: a) hue depending on storage period; b) value (HSV scale) depending on storage period reflecting the brightness of the emitted signal. Devices were stored under vacuum in plastic bags containing an oxygen absorber at $4{ }^{\circ} \mathrm{C}$. 


$$
\text { hue }=\frac{\Delta h u e_{\max }[\text { antibody }]}{c_{50}+[\text { antibody }]}+\text { hue } e_{0}
$$

Equation S1. Equation used for fitting the experimentally obtained response curves (antibody concentration vs. hue) ${ }^{3,4} c_{50}$ indicates the antibody concentration resulting in $50 \%$ of the maximal hue change and $\Delta$ hue $\max _{\max }$

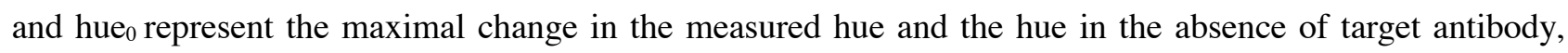
respectively. 
Table S1. Analytical parameters obtained from the response curves shown in Figures 3A and S8.

\begin{tabular}{|c|c|c|c|}
\hline LUMABS type ${ }^{\text {(a) }}$ & hue $_{0}(\text { Degree })^{(b)}$ & $c_{50}(\mathrm{nM})^{(\mathrm{c})}$ & $\begin{array}{l}\text { Mean relative } \\
\text { st. dev. (\%) }{ }^{(d)}\end{array}$ \\
\hline HIV (Figs. 3A, S8) & $171.0 \pm 0.7$ & 23.0 & 8.9 \\
\hline \multicolumn{4}{|l|}{ Batch 1} \\
\hline HA (Fig. 3A) & $168.7 \pm 0.8$ & 10.3 & 8.8 \\
\hline DEN (Fig. 3A) & $165.4 \pm 1.4$ & 70.0 & 5.2 \\
\hline HIV (Fig. S8) & $171.4 \pm 1.2$ & 25.3 & 9.6 \\
\hline \multicolumn{4}{|l|}{ Batch 2} \\
\hline HIV (Fig. S8) & $170.7 \pm 1.8$ & 26.1 & 9.4 \\
\hline Batch 3 & & & \\
\hline
\end{tabular}

(a) The type of LUMABS applied on a single signal detection area; (b) hue in the case of blank blood sample application with standard deviations for measurements of sextuple signals from a single device; (c) antibody concentration resulting in $50 \%$ of the maximal hue change; (d) mean relative standard deviation of hue over the entire response curve divided by the respective maximum $\Delta$ hue difference (hue for blank blood sample and for saturated state). 
Table S2. Analytical parameters obtained from the response curves shown in Figures 6A and 7A.

\begin{tabular}{cccc}
$\begin{array}{c}\text { LUMABS type }^{(\mathrm{a})} \\
\text { hue }\end{array}$ & $c_{50}(\mathrm{nM})^{(\mathrm{c})}$ & $\begin{array}{c}\text { Mean ree })^{(\mathrm{b})} \\
\left.\text { st. dev. }^{(\%)}\right)^{(\mathrm{d})}\end{array}$ \\
\hline $\begin{array}{c}\text { HIV (Fig. 6A) } \\
\text { Digital camera in dark room } \\
\text { HIV (Fig. 6A) }\end{array}$ & $170.7 \pm 1.8$ & 81.3 & 8.9 \\
$\begin{array}{c}\text { Smartphone camera with } \\
\text { adapter }\end{array}$ & $179.7 \pm 2.5$ & 189.8 & 9.0 \\
\\
$\begin{array}{c}\text { HIV-NB-LUMABS } \\
\text { (Fig. 7A) }\end{array}$
\end{tabular}

(a) The type of LUMABS applied on a single signal detection area; (b) hue in the case of blank blood sample application with standard deviations for measurements of sextuple signals from a single device; (c) antibody concentration resulting in 50\% of the maximal hue change; (d) mean relative standard deviation of hue over the entire response curve divided by the respective maximum $\Delta$ hue difference (hue for blank blood sample and for saturated state). 
References

(1) Arts, R.; den Hartog, I.; Zijlema, S. E.; Thijssen, V.; van der Beelen, S. H. E.; Merkx, M. Detection of Antibodies in Blood Plasma Using Bioluminescent Sensor Proteins and a Smartphone. Anal. Chem. 2016, $88(8), 4525-4532$.

(2) Ni, Y.; Arts, R.; Merkx, M. "Ratiometric Bioluminescent Sensor Proteins Based on Intramolecular Split Luciferase Complementation" ACS Sens. 2019, 4 (1), 20-25.

(3) Cantrell, K.; Erenas, M. M.; de Orbe-Payá, I.; Capitán-Vallvey, L. F. Use of the Hue Parameter of the Hue, Saturation, Value Color Space As a Quantitative Analytical Parameter for Bitonal Optical Sensors. Anal. Chem. 2010, 82 (2), 531-542.

(4) Tenda, K.; van Gerven, B.; Arts, R.; Hiruta, Y.; Merkx, M.; Citterio, D. Paper-Based Antibody Detection Devices Using Bioluminescent BRET-Switching Sensor Proteins. Angew. Chem. Int. Ed. 2018, 57 (47), 15369-15373. 